\title{
Perioperative Anaphylaxis to Ethylene Oxide: Twenty Years after, a Diagnosis Revisited
}

\author{
Pascale Dewachter ${ }^{1 *}$, Sonia Tchotourian ${ }^{2}$, Pascale Nicaise-Roland ${ }^{3}$, Catherine Fargeot ${ }^{4}$, Raphaële Escalup ${ }^{5}$, Sylvie Chollet-Martin ${ }^{6}$, Caroline
} Desjardins $^{7}$ and Claudie Mouton-Faivre ${ }^{8}$

${ }^{1}$ Assistance Publique Hôpitaux de Paris, Hôpital Necker-Enfants Malades, Service d'Anesthésie-Réanimation \& SAMU de Paris, Université Paris-Descartes, INSERM U970, Paris, France

${ }^{2}$ Assistance Publique Hôpitaux de Paris, Groupe Hospitalier Pitié-Salpêtrière, Service d'Anesthésie-Réanimation Chirurgicale, Paris, France

${ }^{3}$ Assistance Publique Hôpitaux de Paris, Hôpital Bichat-Claude Bernard, Unité d'Immunologie Auto-immunité \& Hypersensibilités, Paris, France

${ }^{4}$ Assistance Publique Hôpitaux de Paris, Groupe Hospitalier Pitié-Salpêtrière, Service de Pharmacie, Paris, France

${ }^{5}$ Assistance Publique Hôpitaux de Paris, Hôpital Necker-Enfants Malades, Service de Pharmacie, Paris, France

${ }^{6}$ Assistance Publique Hôpitaux de Paris, Hôpital Bichat-Claude Bernard, Unité d'Immunologie Auto-immunité \& Hypersensibilités, Paris \& Université Paris-Sud11,

INSERM UMRS-996, Châtenay-Malabry, France

${ }^{7}$ Assistance Publique Hôpitaux de Paris, Groupe Hospitalier Pitié-Salpêtrière, Service de Pharmacie, Paris, France

${ }^{8}$ Pôle d'Anesthésie-Réanimation Chirurgicale, CHU Hôpital Central, Nancy, France

\begin{abstract}
We report the case of a Spina Bifida patient for whom the diagnosis of combined latex and ethylene oxide-induced anaphylaxis was suggested 20 years ago. Since that time, latex-free environment and gamma-irradiated devices were recommended. A cystotomy was scheduled in 2009 and a new allergological assessment was performed. Latex allergy was confirmed but combined latex and ethylene oxide-induced anaphylaxis was ruled out. Surgery performed in a latexfree environment and by using ethylene oxide sterilized medical devices remained uneventful. As the epidemiology of perioperative anaphylaxis is now supported by a better knowledge, the hypothesis of ethylene oxide-induced anaphylaxis is currently abandoned.
\end{abstract}

\section{Keywords: Anaphylaxis; Latex; Ethylene oxide; Skin tests}

We report the case of a Spina Bifida patient for whom the diagnosis of combined latex and ethylene oxide-induced anaphylaxis was suggested 20 years ago. The diagnosis of latex-induced anaphylaxis possibly associated to an allergy to ethylene oxide was supported by the clinical history associated with positive prick-tests in response to latex as well as positive prick-tests when performed through a piece of latex glove sterilized with ethylene oxide and increased level of specific IgEs against latex. In turn, specific serum IgEs against ethylene oxide remained undetectable. However since that time, latex-free environment and gamma-irradiated devices were recommended for subsequent procedures whereas biomedical devices sterilized with ethylene oxide were excluded in our patient. Twenty-two years after this clinical event, in July 2009, this now 34-year-old man with intestinal neo-bladder was scheduled for a cystotomy for vesical calculi (being then his $33^{\text {rd }}$ surgical procedure). Surgical devices required for the surgery were not only sterilized by irradiation (electron-beam or gamma rays). Indeed, for the last 20 years, sterilization by irradiation has been significantly less used for medical devices, mostly because of its implementation costs and incompatibility with some materials. Thus, a new allergological assessment was scheduled in order to confirm or rule out the diagnosis of ethylene oxide-induced anaphylaxis.

With the patient's consent, skin tests were performed according to standardized procedures $[1,2]$. Prick-tests (PTs) using two different standardized commercial extracts of latex (Stallergènes ${ }^{\circledR}$, Antony, France and Allerbio ${ }^{\circledR}$, Varennes en Argonne, France) were strongly positive within 15 minutes. In turn, PTs performed through a piece of drape sterilized with ethylene oxide, followed by a provocation test (drape sterilized with ethylene oxide that was adherent to a limb and kept in place for an hour), remained negative. The level of specific IgEs against latex (FEIA, ImmunoCAP 250, Phadia SAS,

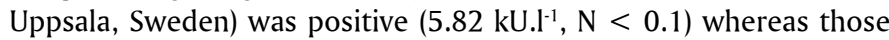
with ethylene oxide, performed by the same technique, remained undetectable. Latex allergy was therefore confirmed according to the clinical history and to the results of allergological assessment. In contrast, ethylene oxide-induced anaphylaxis was ruled out. Surgical procedure was performed in a latex-free environment, and by using ethylene oxide sterilized medical devices. Anesthesia and surgery remained uneventful.

During the past two decades, latex-induced allergy has become a well-recognized condition especially in definite high-risk groups of patients requiring multiple surgical procedures such as Spina Bifida [3]. Concomitantly to the increasing incidence of perioperative latex-induced anaphylaxis, different tools including standardized latex emulsions and quantification of specific IgEs to latex became available in order to prove the diagnosis $[1,2]$. Ethylene oxide is a potent alkalating compound of high chemical reactivity. Ethylene oxide sterilization is mainly used in order to sterilize medical devices or healthcare products that cannot support conventional high temperature steam sterilization. It was previously suggested that ethylene oxide conjugated to albumin might act as an allergen [4]. However, despite the wide patients' exposure to ethylene oxide during the perioperative period, the allergenicity of this compound did not seem to be a major concern, as reports appear to be very rare $[5,6]$. Thus, ethylene oxide-induced anaphylaxis was suggested in 3

*Corresponding author: Pascale Dewachter, Service d'Anesthésie-Réanimation Chirurgicale \& SAMU de Paris, Hôpital Necker-Enfants Malades, AP-HP, Université Paris-Descartes, 149 Rue de Sèvres 75743 Paris, France Cedex, Tel: + 3314449 5407 ; Fax: + 33144495410 ; E-mail: pascale.dewachter@yahoo.fr

Received September 17, 2010; Accepted October 18, 2010; Published October 19, 2010

Citation: Dewachter P, Tchotourian S, Nicaise-Roland P, Fargeot C, Escalup R, et al. (2010) Perioperative Anaphylaxis to Ethylene Oxide: Twenty Years after, a Diagnosis Revisited. J Aller Ther 1:102. doi:10.4172/2155-6121.1000102

Copyright: (C) 2010 Dewachter $P$, et al. This is an open-access article distributed under the terms of the Creative Commons Attribution License, which permits unrestricted use, distribution, and reproduction in any medium, provided the original author and source are credited. 
Citation: Dewachter P, Tchotourian S, Nicaise-Roland P, Fargeot C, Escalup R, et al. (2010) Perioperative Anaphylaxis to Ethylene Oxide: Twenty Years after, a Diagnosis Revisited. J Aller Ther 1:102. doi:10.4172/2155-6121.1000102

Page 2 of 2

patients, on the appearance of "more positive PTs through a latex glove sterilized with ethylene oxide"than through a gamma-irradiated glove [5]. However, differences in skin tests results performed through gloves sterilized by different means may only have reflected a significant variation in latex proteins concentration among these gloves or a closer allergenic community. In two cases out of three [5], the level of serum specific IgEs against ethylene oxide was positive. Nevertheless, the specificity of the IgE-assay to ethylene oxide seems to be poor [7]. In a recent paper reporting the perioperative care of "a patient allergic to ethylene oxide", the proof of the diagnosis was not supported by skin tests to ethylene oxide whereas latexinduced reaction was not ruled out [6]. Allergic reactions to ethylene oxide were reported during hemodialysis session. These immediate reactions occurred during the first use of dialysers and surprisingly not during re-use. Ethylene oxide has therefore been proposed as the main cause of "first-use syndrome" mimicking an anaphylactic reaction [8]. Other reports showed a high percentage of serum ethylene oxide-specific IgE-antibodies in patients having experienced immediate hypersensitivity during hemodialysis [9]. Finally, allergy to ethylene oxide probably plays a part in these reactions but does not explain the whole part [10] whereas some reactions attributed to ethylene oxide are likely to have been thought due to latex [7]. In the present case, we confirmed latex allergy, but ruled out the diagnosis of combined latex and ethylene oxide-induced anaphylaxis. As the diagnosis of ethylene oxide-induced anaphylaxis during the perioperative period has never been formally documented whereas the epidemiology is supported by a better knowledge [3], this hypothesis is currently abandoned. Accordingly, the two latest published guidelines on perioperative anaphylaxis did not mention ethylene oxide-induced reaction $[1,2]$.

In conclusion even 20 years after, it is still time to call into question a previous diagnosis of ethylene oxide-induced anaphylaxis. In our patient, this attitude not only reduced associated medical cost (by avoiding a specific production of irradiated medical devices), but also facilitated care management.

\section{References}

1. Kroigaard M, Garvey LH, Gillberg L, Johansson SG, Mosbech H, et al. (2007) Scandinavian Clinical Practice Guidelines on the diagnosis, management and follow-up of anaphylaxis during anaesthesia. Acta Anaesthesiol Scand 51: 655-670.

2. Harper NJ, Dixon T, Dugue P, Edgar DM, Fay A, et al. (2009) Suspected anaphylactic reactions associated with anaesthesia. Anaesthesia 64: 199211.

3. Dewachter P, Mouton-Faivre C, Emala CW (2009) Anaphylaxis and anesthesia: controversies and new insights. Anesthesiology 111: 1141-1150.

4. Dolovich J, Bell B (1978) Allergy to a product(s) of ethylene oxide gas: demonstration of $\mathrm{IgE}$ and IgG antibodies and hapten specificity. J Allergy Clin Immunol 62: 30-32.

5. Moneret-Vautrin DA, Laxenaire MC, Bavoux F (1990) Allergic shock to latex and ethylene oxide during surgery for spinal bifida. Anesthesiology 73: 556 558

6. Demontoux V, Moreau E, Peyron I, Talon V, Khouri N, et al. (2008) Surgery in a patient allergic to ethylene oxide. Pharm World Sci 30: 208-209.

7. Pittman T, Kiburz J, Steinhardt G, Krock J, Gabriel K (1995) Ethylene oxide allergy in children with spina bifida. J Allergy Clin Immunol 96: 486-488.

8. Ing TS, Ivanovich PT, Daugirdas JT (1987) First-use syndrome and hypersensitivity during hemodialysis: some pieces of the puzzle are falling into place. Artif Organs 11: 79-81.

9. Purello D'Ambrosio F, Savica V, Gangemi S, Ricciardi L, Bagnato GF, et al. (1997) Ethylene oxide allergy in dialysis patients. Nephrol Dial Transplant 12: 1461-1463.

10. Nicholls A (1986) Ethylene oxide and anaphylaxis during haemodialysis. $\mathrm{Br}$ Med J 292: 1221-1222. 